INSTITUT NATIONAL DE RECHERCHE EN INFORMATIQUE ET EN AUTOMATIQUE

\title{
Performance analysis of two sequential Monte Carlo methods and posterior Cramér-Rao bounds for multi-target tracking
}

\author{
Carine Hue — Jean-Pierre Le Cadre — Patrick Pérez
}

$\mathbf{N}^{\circ} 4450$

Avril 2002

THÈME 3 



\title{
Performance analysis of two sequential Monte Carlo methods and posterior Cramér-Rao bounds for multi-target tracking
}

\author{
Carine Hu®, Jean-Pierre Le Cadrđ田, Patrick Pérez \\ Thème 3 - Interaction homme-machine, \\ images, données, connaissances \\ Projet Vista \\ Rapport de recherche $\mathrm{n}^{\circ} 4450$ - Avril $2002-\quad$ 16] pages
}

\begin{abstract}
The multi-target tracking algorithms generally present two basic ingredients: an estimation algorithm coupled with a data association method. In the last years, the use of sequential Monte Carlo methods has grown in many application domains and in particular in target tracking. The state distribution is then estimated with a finite weighted sum of Dirac laws centered around "particles". Very recently, two new algorithms based on sequential Monte Carlo methods have been proposed independently to solve multi-target tracking. The first one solves the data association as in the Joint Probabilistic Data Association (JPDAF) spirit whereas the second uses independent probabilistic assignments. In this paper, we first compare their performance for bearings-only applications. Then, we study how the posterior Cramér-Rao bound, giving a lower bound on the estimation error covariance, can be obtained for multiple targets. Three new bounds are obtained according to the data association assumptions and are evaluated for bearings-only scenarios.
\end{abstract}

Key-words: multi-target tracking, sequential Monte Carlo methods, data association, bearings-only, posterior Cramér-Rao bounds, performance analysis.

\footnotetext{
* IRISA - Université de Rennes 1, Carine.Hue@irisa.fr

$\dagger$ IRISA - CNRS, lecadre@irisa.fr

$\ddagger$ Microsoft Research, Cambridge, pperez@microsoft.com
} 


\section{Analyses de performances de deux algorithmes particulaires et bornes de Cramér-Rao a posteriori pour le suivi multi-cibles.}

Résumé : Les algorithmes de suivi multi-cibles sont en général composés de deux éléments fondamentaux : un algorithme d'estimation couplé avec une méthode d'association des mesures. Ces dernières années, les méthodes d'estimation séquentielles de Monte Carlo ont connu un intérêt grandissant dans de nombreux domaines d'applications y compris pour le pistage. La loi de densité de l'état est alors estimée par une somme finie pondérée de lois de Dirac centrées en des "particules". Fondés sur ces méthodes particulaires, deux nouveaux algorithmes ont très récemment été indépendamment proposées pour le suivi multi-cibles. Le premier traite l'association de données avec une approche semblable à celle du Joint Probabilistic Data Association Filter (JPDAF) alors que le second introduit des probabilités d'association. Dans cet article, nous comparons tout d'abord les performances de ces deux algorithmes dans un contexte de pistage par mesure d'angles. Nous étudions ensuite l'extension de l'expression de la borne de Cramér-Rao a posteriori au problème d'estimation multi-cibles, afin d'obtenir une borne minimale sur la covariance de l'erreur d'estimation. Trois expressions analytiques sont en réalité obtenues selon les hypothèses d'association émises. Elles sont enfin évaluées pour le pistage.

Mots-clés : suivi multi-cibles, méthodes séquentielles de Monte Carlo, association de données, pistage par mesure d'angles, bornes de Cramér-Rao a posteriori, analyse de performances. 


\section{Introduction}

For a long time, Kalman filters and extended Kalman filters were the classical solutions for estimation problems. By filtering the two first moments of the state distribution, they provide an optimal solution only if the dynamic and measurement models are Gaussian and linear. When tracking not one single but multiple targets, the difficulty lies on the fact that the estimation of the targets and the association between the measurements and the targets must be solved jointly. Existing multi-target tracking algorithms generally present two basic ingredients: an estimation algorithm coupled with a data association method. Among the most popular algorithms based on (extended) Kalman filters, are the JPDAF [1], the Multiple Hypothesis Tracker (мнт) [2] or the Probabilisitc мнт (рмнт) [3], 4]. They vary on the association method used. With the development of the sequential Monte Carlo (SMC) methods, new opportunities for multi-target tracking have appeared. The state distribution is then estimated with a finite weighted sum of Dirac laws centered around "particles". This work presents and compares two algorithms, proposed independently. They are close in the sense that they use in the same way the SMC methods. In Section 2, this common framework is first described. Then the two algorithms so-called SIR-JPDA and MOPF are presented. Section 3 is dedicated to the comparison of the performance of both algorithms in simulated scenarios using bearingsonly observations. In Section 4, the definition and the derivation of the posterior Cramér-Rao bound are recalled and recursively expressed for single target filtering. Then the extension of such a bound to the case of multiple target filtering is studied: three bounds are in fact derived depending on the association assumptions. They are evaluated by Monte Carlo simulations.

\section{The MTT problem in the light of SMC methods}

\subsection{General framework}

Let $M$ be the number of targets to track. This number is assumed to be known and fixed for the moment. The index $i$ designates one among the $M$ targets and is always used as superscript. Multitarget tracking consists in estimating the state vector made by concatenating the state vectors of all targets. It is generally assumed that the targets are moving according to independent Markovian dynamics. At time $t, X_{t}=\left(X_{t}^{1}, \ldots, X_{t}^{M}\right)$ follows the state equation decomposed in $M$ partial equations:

$$
X_{t}^{i}=F_{t}^{i}\left(X_{t-1}^{i}, V_{t}^{i}\right) \quad \forall i=1, \ldots, M .
$$

The noises $\left(V_{t}^{i}\right)$ and $\left(V_{t}^{i^{\prime}}\right)$ are supposed only to be white both temporally and spatially, and independent for $i \neq i^{\prime}$.

The observation vector collected at time $t$ is denoted by $y_{t}=\left(y_{t}^{1}, \ldots, y_{t}^{m_{t}}\right)$. The index $j$ is used as first superscript to refer to one of the $m_{t}$ measurements. The vector $y_{t}$ is composed of detection measurements and clutter measurements. The false alarms are assumed to be uniformly distributed in the observation area. Their number is assumed to arise from a Poisson density of parameter $\lambda V$ where $V$ is the volume of the observation area and $\lambda$ the number of false alarms per unit volume. As we do not know the origin of each measurement, one has to introduce the vector $K_{t}$ to describe the associations between the measurements and the targets. Each component $K_{t}^{j}$ is a random variable that takes its values among $\{0, \ldots, M\}$. Thus, $K_{t}^{j}=i$ indicates that $y_{t}^{j}$ is associated with the $i$ th target. In this case, $y_{t}^{j}$ is a realization of the stochastic process:

$$
Y_{t}^{j}=H_{t}^{i}\left(X_{t}^{i}, W_{t}^{j}\right) \text { if } K_{t}^{j}=i
$$

Again, the noises $\left(W_{t}^{j}\right)$ and $\left(W_{t}^{j^{\prime}}\right)$ are supposed only to be white noises, independent for $j \neq j^{\prime}$. We assume that the functions $H_{t}^{i}$ are such that they can be associated to functional forms $l_{t}^{i}$ such that

$l_{t}^{i}(y ; x) \propto p\left(Y_{t}^{j}=y \mid K_{t}^{j}=i, X_{t}^{i}=x\right)$.

We dedicate the model 0 to false alarms. Thus, if $K_{t}^{j}=0$, the $j$ th measurement is associated to the clutter, but we do not associate any kinematic model to false alarms. As the indexing of the measurements is arbitrary, all the measurements have the same a priori probability to be associated with a given model $i$. At time $t$, these association probabilities define the vector $\pi_{t}=\left(\pi_{t}^{0}, \pi_{t}^{1}, \ldots, \pi_{t}^{M}\right) \in[0,1]^{M+1}$. Thus, for 
$i=1, \ldots, M, \pi_{t}^{i} \triangleq \mathbb{P}\left(K_{t}^{j}=i\right)$ for all $j=1, \ldots, m_{t}$ is the discrete probability that any measurement is associated with the $i$ th target.

To solve the data association some assumptions are commonly made [5]:

(A1) One measurement can originate from one target or from the clutter.

(A2) One target can produce zero or one measurement at one time.

(A3) One target can produce zero or several measurements at one time.

The assumption (A1) expresses that the association is exclusive and exhaustive. Consequently, $\sum_{i=0}^{M} \pi_{t}^{i}=1$. The assumption (A2) implies that $m_{t}$ may differ from $M$ and above all that the association variables $K_{t}^{j}$ for $j=1, \ldots, m_{t}$ are dependent.

The assumption (A3) is often criticized because it may not match the physical reality. However, from a mathematical point of view it ensures the stochastic independence of the variables $K_{t}^{j}$ and it drastically reduces the complexity of the $\pi_{t}$ vector estimation.

In the context of multitarget tracking, particle filters are appealing: as the association needs only to be considered at a given time iteration, the complexity of data association is reduced. For a state of art of the proposed algorithms the reader can refer to [6]. In the two following methods, particle dimension is the sum of those of the individual state spaces corresponding to each target. Each of these concatenated vectors then gives jointly a representation of all targets. The initial particle set $S_{0}=\left(s_{0}^{n}, 1 / N\right)_{n=1, \ldots, N}$ is such that each component $s_{0}^{n, i}$ for $i=1, \ldots, M$ is sampled from $p\left(X_{0}^{i}\right)$ independently from the others. Assume we have obtained $S_{t-1}=\left(s_{t-1}^{n}, q_{t-1}^{n}\right)_{n=1, \ldots, N}$ with $\sum_{n=1}^{N} q_{t-1}^{n}=1$. Each particle is a vector of dimension $\sum_{i=1}^{M} n_{x}^{i}$ where we denote by $s_{t-1}^{n, i}$ the $i$ th component of $s_{t-1}^{n}$ and where $n_{x}^{i}$ designates the dimension of object $i$. The prediction can be done according to the following equation:

$$
\text { For } n=1, \ldots, N \tilde{s}_{t}^{n}=\left(\begin{array}{c}
F_{t}^{1}\left(s_{t-1}^{n, 1}, v_{t}^{n, 1}\right) \\
\vdots \\
F_{t}^{M}\left(s_{t-1}^{n, M}, v_{t}^{n, M}\right)
\end{array}\right)
$$

where $v_{t}^{n, i}$ are independent realizations of $V_{t}^{i}$. The computation of the likelihood of the observations conditioned by the $n$th particle differs according to the association assumptions and enables to update the weights $q_{t}^{n}$ from $q_{t-1}^{n}$. The set $\left\{s_{t}^{n}\right\}$ is finally obtained by resampling $\left\{\tilde{s}_{t}^{n}\right\}$ according the weight set $\left\{q_{t}^{n}\right\}$ or is equal to $\left\{\tilde{s}_{t}^{n}\right\}$ if resampling is not necessary.

\subsection{The MOPF}

Under (A1) and (A3), the components of the association vector $K_{t}$ are independent and we can write:

$$
\begin{aligned}
& p\left(Y_{t}=\left(y_{t}^{1}, \ldots, y_{t}^{m_{t}}\right) \mid X_{t}=\tilde{s}_{t}^{n}\right) \stackrel{A 1}{=}{ }^{A 3} \prod_{j=1}^{m_{t}} p\left(y_{t}^{j} \mid \tilde{s}_{t}^{n}\right) \\
& \propto \prod_{j=1}^{m_{t}}\left[\frac{\pi_{t}^{0}}{V}+\sum_{i=1}^{M} l_{t}^{i}\left(y_{t}^{j} ; \tilde{s}_{t}^{n, i}\right) \pi_{t}^{i}\right] .
\end{aligned}
$$

Estimation of $\pi_{t}$ is needed and is computed with a Gibbs sampler in the MOPF. For details on this algorithm, the reader is referred to [6]. 
- Initialization: $\left\{\begin{array}{l}s_{0}^{n} \sim p\left(X_{0}\right) \\ q_{0}^{n}=1 / N\end{array} \quad n=1, \ldots, N\right.$.

- For $t=1, \ldots, T$ :

○ Prediction: $\left\{\begin{array}{l}v_{t}^{n} \sim p\left(V_{t}\right) \\ \tilde{s}_{t}^{n}=F_{t}\left(s_{t-1}^{n}, v_{t}^{n}\right)\end{array} \quad n=1, \ldots, N\right.$.

$\circ$ Weighting

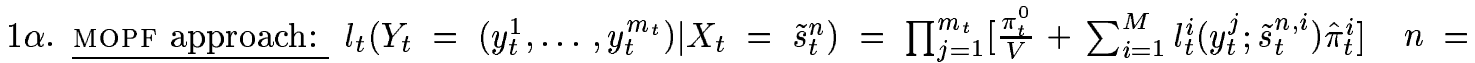

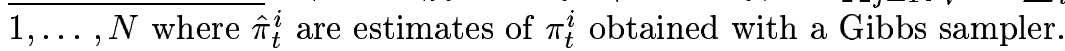

1 $\beta$. SIR-JPDA approach: $l_{t}\left(Y_{t}=\left(y_{t}^{1}, \ldots, y_{t}^{m_{t}}\right) \mid X_{t}=\tilde{s}_{t}^{n}\right)=\sum_{k_{t}} \prod_{j=1}^{m_{t}} p\left(y_{t}^{j} \mid \tilde{s}_{t}^{n}, k_{t}\right) p\left(k_{t}\right)$ where $p\left(k_{t}\right)$ is computed according to (6).

2. $q_{t}^{n} \propto q_{t-1}^{n} l_{t}\left(y_{t} \mid \tilde{s}_{t}^{n}\right) \quad n=1, \ldots, N$.

○ Return $\widehat{\mathbb{E}} g\left(X_{t}\right)=\sum_{n=1}^{N} q_{t}^{n} g\left(\tilde{s}_{t}^{n}\right)$.

$\circ \hat{N}_{e f f}=\frac{1}{\sum_{n=1}^{N}\left(q_{t}^{n}\right)^{2}}$.

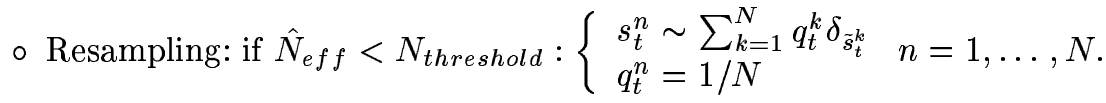

Figure 1: The MOPF and the SIR-JPDA algorithms for multiple objects with adaptive resampling.

\subsection{The SIR-JPDA algorithm}

This approach has initially been used in [7] and [8]. The likelihood is decomposed in a sum over all the possible values of the association vector $K_{t}$ assuming the dependence of its components (A2):

$$
\begin{aligned}
& p\left(Y_{t}=\left(y_{t}^{1}, \ldots, y_{t}^{m_{t}}\right) \mid X_{t}=\tilde{s}_{t}^{n}\right) \\
& =\sum_{k_{t}} p\left(Y_{t}=\left(y_{t}^{1}, \ldots, y_{t}^{m_{t}}\right) \mid X_{t}=\tilde{s}_{t}^{n}, K_{t}=k_{t}\right) p\left(K_{t}=k_{t}\right) \\
& =\sum_{k_{t}} \prod_{j=1}^{m_{t}} p\left(y_{t}^{j} \mid \tilde{s}_{t}^{n}, k_{t}\right) p\left(k_{t}\right) .
\end{aligned}
$$

The associations between the measurements and the targets are established under the assumptions (A1)-(A2) exposed in Section 2.1] The a priori probability of each association vector $k_{t}$ is:

$$
p\left(k_{t}\right)=\frac{\Phi^{k_{t}} !}{m_{t} !} p_{F}\left(\Phi^{k_{t}}\right) \prod_{i=1}^{M} P_{d}^{D^{k_{t}}(i)} \prod_{i=1}^{M}\left(1-P_{d}\right)^{1-D^{k_{t}}(i)}
$$

where $\Phi^{k_{t}}$ is the number of false alarms in $k_{t}, p_{F}\left(\Phi^{k_{t}}\right)$ the probability to have $\Phi^{k_{t}}$ false alarms, $P_{d}$ the probability for a target to be detected and $D^{k_{t}}(i)=\{1,0\}$ depending on whether target $i$ is detected or not. Both algorithms are presented in a common structure in Fig 1. We have compared the performance of the MOPF and of the SIR-JPDA in the following experiments.

\section{Simulation results}

We deal with classical bearings-only experiments with three targets. In the context of a slowly maneuvering target, we have chosen a nearly-constant-velocity model. 

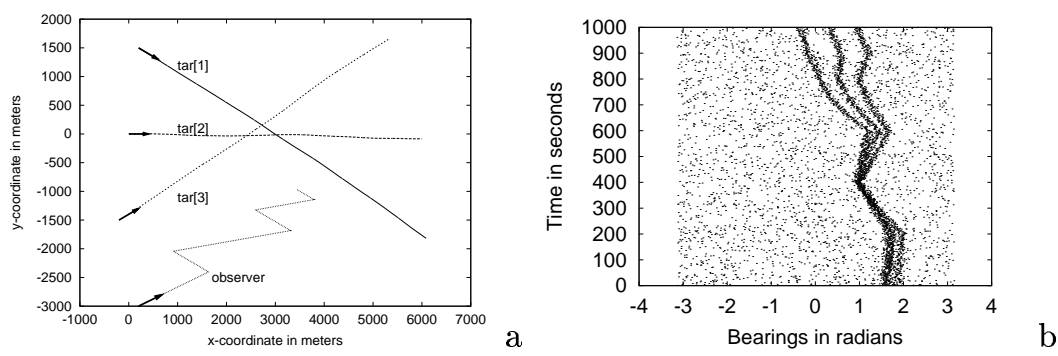

Figure 2: (a) Trajectories of the three targets and of the observer; (b) Measurements simulated with $P_{d}=0.9$ and $\lambda V=3$.
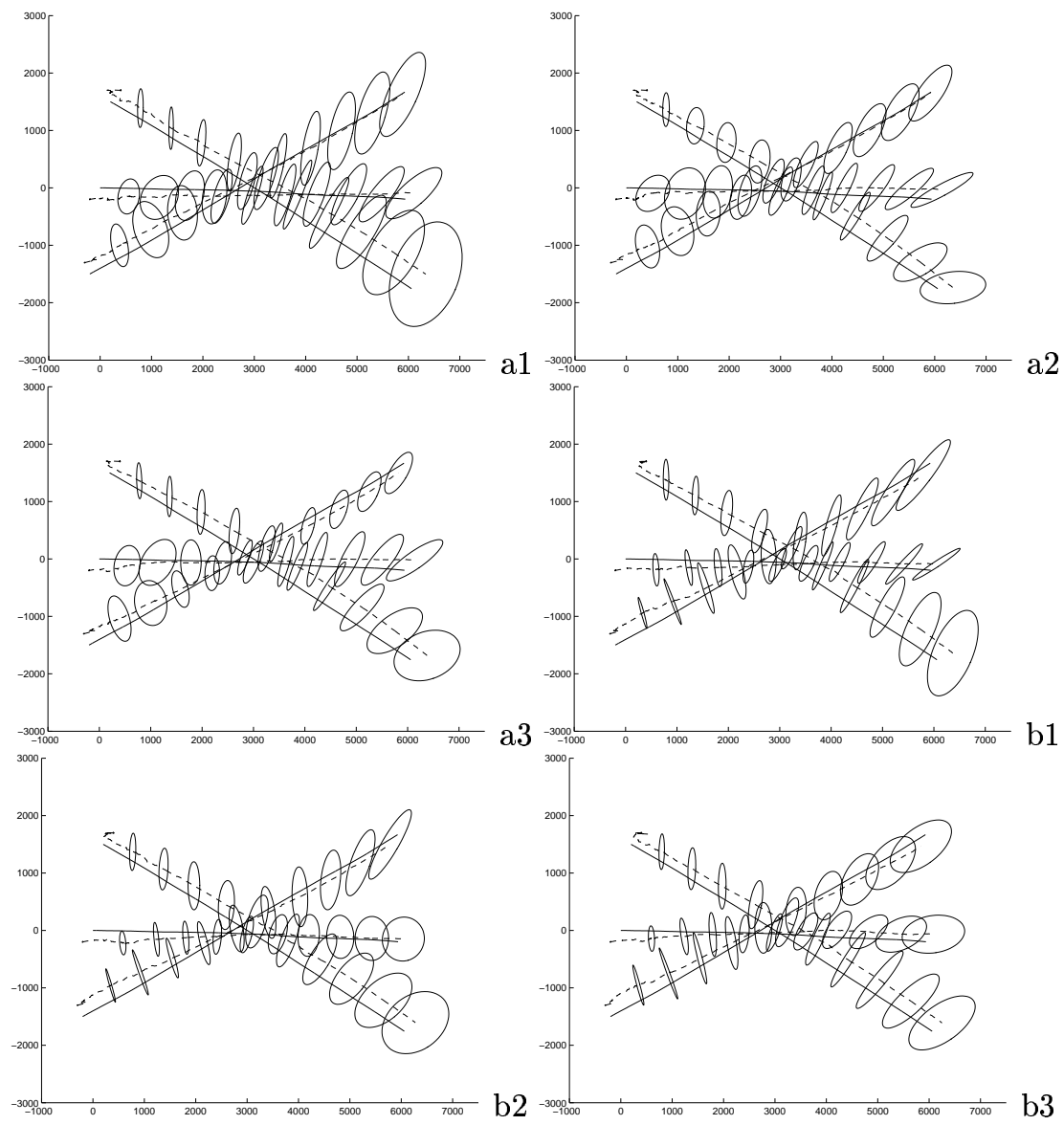

Figure 3: Averaged estimates (dotted lines) and $2 \sigma$ confidence ellipses with 2000 particles; Top row: SIR-JPDA; Bottom row: MOPF;(a1)-(b1) $\lambda V=1$; (a2)-(b2) $\lambda V=2$; (a3)-(b3) $\lambda V=3$. 


\section{$3.1 \quad$ The model}

The state vector $X_{t}^{i}$ represents the coordinates and the velocities in the $x-y$ plane: $X_{t}^{i}=\left(x_{t}^{i}, y_{t}^{i}, v x_{t}^{i}, v y_{t}^{i}\right)$ for $i=1,2,3$. For each target, the discretized state equation associated with time period $\Delta t$ is:

$$
X_{t+\Delta t}^{i}=\left(\begin{array}{ll}
I_{2} & \Delta t I_{2} \\
0 & I_{2}
\end{array}\right) X_{t}^{i}+\left(\begin{array}{ll}
\frac{\Delta t^{2}}{2} I_{2} & 0 \\
0 & \Delta t I_{2}
\end{array}\right) V_{t}^{i}
$$

where $I_{2}$ is the identity matrix in dimension 2 and $V_{t}^{i}$ is a Gaussian zero-mean vector with covariance matrix $\Sigma_{V}=\operatorname{diag}\left[\sigma_{x}^{2}, \sigma_{y}^{2}, \sigma_{x}^{2}, \sigma_{y}^{2}\right]$. A set of $m_{t}$ measurements is available at discrete times and can be divided in two subsets:

- A subset of "true" measurements which follow [8]. A measurement produced by the $i$ th target is generated according to:

$$
Y_{t}^{j}=\arctan \left(\frac{y_{t}^{i}-y_{t}^{o b s}}{x_{t}^{i}-x_{t}^{o b s}}\right)+W_{t}^{j}
$$

where $W_{t}^{j}$ is a zero-mean Gaussian noise with covariance $\sigma_{w}^{2}$ independent of $V_{t}$, and $x_{o b s}$ and $y_{o b s}$ are the Cartesian coordinates of the observer, which are known. We assume that the measurement produced by one target is available with a detection probability $P_{d}$.

- A subset of "false" measurements whose number follows a Poisson distribution with mean $\lambda V$ where $\lambda$ is the mean number of false alarms per unit volume. We assume these false alarms are independent and uniformly distributed within the observation volume $V$.

The initial coordinates of the targets and of the observer are the following (in meter and meter/second resp.):

$$
\begin{aligned}
& X_{0}^{1}=(200,1500,1,-0.5)^{T} ; X_{0}^{2}=(0,0,1,0)^{T} ; \\
& X_{0}^{3}=(-200,-1500,1,0.5)^{T} ; \\
& X_{0}^{\text {obs }}=(200,-3000,1.2,0.5)^{T} .
\end{aligned}
$$

The dynamic noise is a normal zero-mean Gaussian vector with $\sigma_{x}=\sigma_{y}=0.0005 \mathrm{~ms}^{-1}$. We use the same dynamic noise to predict the particle. The observer is following a leg by leg trajectory. Its velocity vector is constant on each leg and modified at the following instants, so that:

$$
\begin{aligned}
& \left(\begin{array}{l}
v x_{200,600,900}^{o b s} \\
v y_{200,600,900}^{o b s}
\end{array}\right)=\left(\begin{array}{c}
-0.6 \\
0.3
\end{array}\right) ; \\
& \left(\begin{array}{l}
v x_{400,800}^{o b s} \\
v y_{400,800}^{o b s}
\end{array}\right)=\left(\begin{array}{c}
2.0 \\
0.3
\end{array}\right) ;
\end{aligned}
$$

The trajectories of the three objects and of the observer are plotted in Fig. [2]a.

\subsection{Results of the experiments}

In the following experiments, the detection probability $P_{d}$ is fixed to 0.9 and $\lambda V=1,2,3$. For $\lambda V=3$, the obtained simulated bearings are plotted in Fig. 2 $\mathrm{b}$. We have first compared the computational cost for

Table 1: Computational cost for one iteration on a 863 Mhz Pentium III.

\begin{tabular}{|l|c|c|c|}
\hline clutter density & 1 & 2 & 3 \\
\hline SIR-JPDA & $155 \mathrm{~ms}$ & $290 \mathrm{~ms}$ & $540 \mathrm{~ms}$ \\
\hline MOPF & $400 \mathrm{~ms}$ & $415 \mathrm{~ms}$ & $430 \mathrm{~ms}$ \\
\hline
\end{tabular}

one iteration of each algorithm according to the total number of measurements $m_{t}$. Table 1 contains those costs according to the density clutter. The increase is almost exponential for the SIR-JPDA and linear for the MOPF. One SIR-JPDA iteration, with the exhaustive enumeration of all the possible associations, becomes 
longer than a MOPF iteration as soon as there is in average 6 measurements every instant $(\lambda V=3)$. This constitutes a real drawback of the SIR-JPDA for experiments with high values of $m_{t}$. The initialization of the particle set has been done for both algorithms according to a Gaussian law whose mean vector $X_{\text {mean }}$ and covariance matrix $X_{c o v}$ are:

$$
\begin{aligned}
& X_{\text {mean }}^{1}=X_{0}^{1}+(200,200,0,0)^{T} ; \\
& X_{\text {mean }}^{2}=X_{0}^{2}+(-200,-200,0,0)^{T} ; \\
& X_{\text {mean }}^{3}=X_{0}^{3}+(-100,200,0,0)^{T} ; \\
& X_{\text {cov }}^{i}=\operatorname{diag}(150,150,0.1,0.1) \text { for } i=1,2,3 .
\end{aligned}
$$

We have performed 20 runs of each algorithm for $\lambda V=1,2,3$. The true trajectories and the mean estimates over these 20 runs are plotted in Fig. 3 a-f. The confidence ellipses represent the variance on position over 20 runs of the posterior mean estimates and enable to assess the variance of the MTPF and SIR-JPDA estimator for $N=2000$ particles. The mean estimates are similar whatever the clutter density. However, for these experiments, the confidence ellipses obtained with the SIR-JPDA are larger than those obtained with the MOPF. To compare more generally the algorithms, we propose to study the posterior Cramér-Rao bounds of such estimation problems.

\section{Posterior Cramér-Rao bounds for multi-target tracking}

The two presented algorithms are suboptimal: first the particle number is finite. Second, the Mopf needs the $\pi_{t}$ estimation.

It is of great interest to derive minimum variance bounds on estimation errors to have an idea of the maximum knowledge on the states that can be expected and to assess the quality of the results of the proposed algorithms compared to the bounds. First defined and used in the context of constant parameter estimation, the inverse of the Fisher information matrix, commonly called the Cramér-Rao (CR) bound has been extended to the case of random parameter estimation in [9], then called the posterior Cramér-Rao bound (PCRB). Let $X \in \mathbb{R}^{n_{x}}$ be a stochastic vector and $Y \in \mathbb{R}^{n_{y}}$ a stochastic observation vector. The mean-square error of any estimate $\hat{X}(Y)$ satisfies the inequality

$$
\mathbb{E}(\hat{X}(Y)-X)(\hat{X}(Y)-X)^{T} \geq J^{-1}
$$

where $J=-\mathbb{E}\left[\frac{\partial^{2} \log p_{X, Y}(X, Y)}{\partial X^{2}}\right]$ is the Fisher information matrix and where the expectations are w.r.t. the joint density $p_{X, Y}(X, Y)$ under the following conditions:

- $\frac{\partial p_{X, Y}(X, Y)}{\partial X}$ and $\frac{\partial^{2} p_{X, Y}(X, Y)}{\partial X^{2}}$ exist and are absolutely integrable w.r.t. $X$ and $Y$.

- the estimator bias

$$
\begin{gathered}
\qquad B(X)=\int_{\mathbb{R}^{n_{y}}}(\hat{X}(Y)-X) p_{Y \mid X}(Y \mid X) d Y \\
\text { satisfies: } \lim _{X_{l} \rightarrow \pm \infty} B(X) p(X)=0, \forall l=1, \ldots, n_{x} .
\end{gathered}
$$

Let us define the non-linear filtering problem by the following system:

$$
\left\{\begin{array}{l}
X_{t}=F_{t}\left(X_{t-1}, V_{t}\right) \\
Y_{t}=H_{t}\left(X_{t}, W_{t}\right)
\end{array}\right.
$$

and denote $X_{0: t} \triangleq\left(X_{0}, \ldots, X_{t}\right)$ and $Y_{0: t} \triangleq\left(Y_{0}, \ldots, Y_{t}\right)$. Using the notations of [10], $J\left(X_{0: t}\right)$ denotes the $\left((t+1) n_{x} \times(t+1) n_{x}\right)$ information matrix of $X_{0: t}$ and $J_{X_{t}}$ denotes the $n_{x} \times n_{x}$ information submatrix of $X_{t}$ which is the inverse of the $n_{x} \times n_{x}$ right-lower block of $\left[J\left(X_{0: t}\right)\right]^{-1}$. To avoid inversion of too large matrices, a recursive expression of the bound $J_{X_{t}}$ has been presented recently in [10], [11, and summarized by the following formula:

$$
J_{X_{t+1}}=D_{X_{t}}^{22}-D_{X_{t}}^{21}\left(J_{X_{t}}+D_{X_{t}}^{11}\right)^{-1} D_{X_{t}}^{12}
$$


where

$$
\begin{aligned}
& D_{X_{t}}^{11}=\mathbb{E}\left[-\Delta_{X_{t}}^{X_{t}} \log p\left(X_{t+1} \mid X_{t}\right)\right] \\
& D_{X_{t}}^{12}=\mathbb{E}\left[-\Delta_{X_{t}}^{X_{t+1}} \log p\left(X_{t+1} \mid X_{t}\right)\right] \\
& D_{X_{t}}^{21}=\mathbb{E}\left[-\Delta_{X_{t+1}}^{X_{t}} \log p\left(X_{t+1} \mid X_{t}\right)\right]=\left[D_{X_{t}}^{12}\right]^{T} \\
& D_{X_{t}}^{22}=\mathbb{E}\left[-\Delta_{X_{t+1}}^{X_{t+1}} \log p\left(X_{t+1} \mid X_{t}\right)\right]+\mathbb{E}\left[-\Delta_{X_{t+1}}^{X_{t+1}} \log p\left(Y_{t+1} \mid X_{t+1}\right)\right]
\end{aligned}
$$

and where the $\nabla$ and $\Delta$ operators denote the first and second partial derivatives respectively:

$$
\nabla_{X}=\left[\frac{\partial}{\partial_{x_{1}}}, \ldots, \frac{\partial}{\partial_{x_{n_{x}}}}\right]^{T} ; \Delta_{X}^{Y}=\nabla_{X} \nabla_{Y}^{T}
$$

The matrix $J_{X_{t}+1}^{-1}$ provide a lower bound on the mean-square error of estimating $X_{t+1}$. Recently, the extension of these bounds to the case of linear and non-linear filtering with measurement origin uncertainty due to clutter has been studied in [12] and [13]. It mainly consists of replacing the classic pdf of the measurement given the state by the pdf of the measurement vector taking into account the measurement uncertainty. The new PCRB obtained then appears as multiplied by an information reduction factor.

Now, let us see how these results can be extended and used in the case of multiple target filtering defined by (11) and (2). Note that in this case, the measurement vector is composed of detection measurements issued from the different targets and of false alarms. The following extension then takes into account the measurement uncertainty and the extension of one to multiple targets. First, the recursive Equation (15) can be obtained as well for multiple targets using the structure of the joint law :

$$
p\left(X_{0: t+1}, Y_{0: t+1}\right)=p\left(X_{0: t}, Y_{0: t}\right) p\left(X_{t+1} \mid X_{t}\right) p\left(Y_{t+1} \mid X_{t+1}\right) .
$$

This structure is still true for multiple targets, which leads to the same recursive formula for the information matrix. As the targets are supposed to move according independent dynamics, we have

$$
\log p\left(X_{t+1}^{1: M} \mid X_{t}^{1: M}\right)=\sum_{i=1}^{M} \log p\left(X_{t+1}^{i} \mid X_{t}^{i}\right) .
$$

Consequently, the matrices $D_{X_{t}}^{11}, D_{X_{t}}^{12}$ and the first term of $D_{X_{t}}^{22}$ are simply block-diagonal matrices where the

$i$ th block is computed w.r.t. $X_{t}^{i}$ and $X_{t+1}^{i}$. To evaluate the second term of $D_{X_{t}}^{22}$, i.e., $\mathbb{E}\left[-\Delta_{X_{t+1}^{1: 1}}^{X_{t+1}^{1: M}} \log p\left(Y_{t+1} \mid X_{t+1}^{1: M}\right)\right]$, we are confronted again with the association problem: some additionnal hypotheses must be formulated to write further the likelihood $p\left(Y_{t+1} \mid X_{t+1}\right)$. The problem is that the hypotheses depend on the estimation algorithm and should not influence the theoritical bound. We propose to derive three bounds:

$B^{1}$, the PCRB computed under the $(A 1)$ and $(A 2)$ assumptions.

$B^{2}$, the PCRB computed under the MOPF assumptions, and in particular the $(A 1)$ and $(A 3)$ assumptions.

$B^{3}$, the PRCB computed under the assumption that the associations are known.

The evaluation of these three bounds will allow to measure the influence of theses asumptions and to evaluate the performance of the SIR-JPDA and the MOPF compared with $B^{1}$ and $B^{2}$ respectively.

\subsection{PCRB B1}

We can write:

$$
\begin{aligned}
& \log p\left(Y_{t}=y_{t} \mid X_{t}=x_{t}\right) \\
& \stackrel{A 1}{=}{ }^{A 2} \log \sum_{k_{t}} p\left(y_{t}=\left(y_{t}^{1}, \ldots, y_{t}^{m_{t}}\right) \mid x_{t}, k_{t}\right) p\left(k_{t}\right) \\
& =\log \sum_{k_{t}} \prod_{j=1}^{m_{t}} p\left(y_{t}^{j} \mid x_{t}, k_{t}\right) p\left(k_{t}\right) .
\end{aligned}
$$


The gradient of the log-likelihood w.r.t. $X_{t}^{i}$ is:

$$
\nabla_{X_{t}^{i}} \log p\left(y_{t} \mid x_{t}\right)=\frac{\sum_{k_{t}} \nabla_{X_{t}^{i}} \prod_{j=1}^{m_{t}} p\left(y_{t}^{j} \mid x_{t}, k_{t}\right) p\left(k_{t}\right)}{p\left(y_{t} \mid x_{t}\right)} .
$$

Let denote by $k_{t} \supset i$ the associations that associate one measurement to the $i$ th target. Under (A2), there exists at most one such measurement, denoted $j^{i}$. Then,

$$
\nabla_{X_{t}^{i}} \log p\left(y_{t} \mid x_{t}\right)=\frac{\sum_{k_{t} \supset i} \prod_{j \neq j^{i}} p\left(y_{t}^{j} \mid x_{t}, k_{t}\right) p\left(k_{t}\right) \nabla_{X_{t}^{i}} p\left(y_{t}^{j^{i}} \mid x_{t}^{i}\right)}{p\left(y_{t} \mid x_{t}\right)} .
$$

After some computations, we obtain for all $i 1, i 2=1, \ldots, M$ :

$\mathbb{E}\left[-\Delta_{X_{t}^{i 1}}^{X_{1}^{i 2}} \log p\left(Y_{t} \mid X_{t}\right)\right]=$

$\mathbb{E}_{X_{t}} \mathbb{E}_{Y_{t} \mid X_{t}}\left[\frac{\sum_{k_{t} \supset i 1} \prod_{j \neq j^{i 1}} p\left(y_{t}^{j} \mid x_{t}, k_{t}\right) p\left(k_{t}\right) \nabla_{X_{t}^{i 1}} p\left(y_{t}^{j^{i 1}} \mid x_{t}^{i 1}\right)}{p\left(y_{t} \mid x_{t}\right)^{2}} \cdot \sum_{k_{t} \supset i 2} \prod_{j \neq j^{i 2}} p\left(y_{t}^{j} \mid x_{t}, k_{t}\right) p\left(k_{t}\right)\left(\nabla_{X_{t}^{i 2}} p\left(y_{t}^{j^{i 2}} \mid x_{t}^{i 2}\right)\right)^{T}\right]$

where $\mathbb{E}_{X_{t}}$ and $\mathbb{E}_{Y_{t} \mid X_{t}}$ denote resp. the expectation w.r.t. the density $p\left(X_{t}\right)$ and $p\left(Y_{t} \mid X_{t}\right)$. Let us notice that the integrals w.r.t. $y_{t}$ are $m_{t} \times n_{y}$ dimensional.

\subsection{PCRB B2}

The stochastic vector to be estimated contains now the association vector $\pi$. As it satisfies the equality $\sum_{i=0}^{M} \pi_{t}^{i}=1$ and as $\pi_{t}^{0}$ is fixed at each instant, we only consider the $M-1$ components $\Pi_{t}^{1: M-1}=$ $\left(\Pi_{t}^{1}, \ldots, \Pi_{t}^{M-1}\right)$. Let us define $\Phi_{t}=\left(\Pi_{t}^{1: M-1}, X_{t}^{1: M}\right)$, the joint law is:

$$
p_{t+1} \triangleq p\left(\Phi_{0: t+1}, Y_{0: t+1}\right)=p_{t} \cdot p\left(Y_{t+1} \mid \Phi_{t+1}\right) p\left(X_{t+1} \mid X_{t}\right) p\left(\Pi_{t+1}\right) .
$$

Let $J\left(\Phi_{0: t}\right)$ be the information matrix of $\Phi_{0: t}$ associated with $p_{t}$, we are interested in a recursive expression on $t$ of the information submatrix $J_{\Phi_{t}}$ for estimating $\Phi_{t}$. Let us define the following notation: for two vectors $\alpha, \beta$ and $p$ a probability law,

$$
J_{\alpha}^{\beta}(p) \triangleq \mathbb{E}\left[-\Delta_{\alpha}^{\beta} \log (p)\right] .
$$

Using the structure of the joint law $p_{t+1}$ and the same argument as in [10, the following recursive formula can be shown (see the proof in Appendix):

$$
J_{\Phi_{t+1}}=D_{\Phi_{t}}^{22}-D_{\Phi_{t}}^{21}\left(J_{\Phi_{t}}+D_{\Phi_{t}}^{11}\right)^{-1} D_{\Phi_{t}}^{12}
$$

where

$$
\begin{aligned}
D_{\Phi_{t}}^{11} & =J_{\Phi_{t}}^{\Phi_{t}}\left(p\left(X_{t+1} \mid X_{t}\right)\right)=\left[\begin{array}{cc}
0 & 0 \\
0 & D_{X_{t}}^{11}
\end{array}\right] ; \\
D_{\Phi_{t}}^{12} & =J_{\Phi_{t}}^{\Phi_{t+1}}\left(p\left(X_{t+1} \mid X_{t}\right)\right)=\left[\begin{array}{cc}
0 & 0 \\
0 & D_{X_{t}}^{12}
\end{array}\right] ; \\
D_{\Phi_{t}}^{22} & =J_{\Phi_{t+1}}^{\Phi_{t+1}}\left(p\left(Y_{t+1} \mid \Phi_{t+1}\right) p\left(X_{t+1} \mid X_{t}\right) p\left(\Pi_{t+1}\right)\right) \\
& =\left[\begin{array}{cc}
0 & 0 \\
0 & J_{X_{t+1}}^{X_{t+1}}\left(p\left(X_{t+1} \mid X_{t}\right)\right)
\end{array}\right]+\left[\begin{array}{cc}
J_{\Pi_{t+1}}^{\Pi_{t+1}}\left(p\left(\Pi_{t+1}\right)\right) & 0 \\
0 & 0
\end{array}\right]+J_{\Phi_{t+1}}^{\Phi_{t+1}}\left(p\left(Y_{t+1} \mid \Phi_{t+1}\right)\right) .
\end{aligned}
$$

Once $J_{\Phi_{t}}$ is recursively computed, a lower bound on the mean square error of estimating $X_{t}$ is given by inversion formula applied to the right lower block $J_{X_{t}}$ of $J_{\Phi_{t}}=\left[\begin{array}{cc}J_{\Pi_{t}} & J_{\Pi_{t}}^{X_{t}} \\ J_{X_{t}}^{\Pi_{t}} & J_{X_{t}}\end{array}\right]$ :

$$
\mathbb{E}(\hat{X}(Y)-X)(\hat{X}(Y)-X)^{T} \geq\left[J_{X_{t}}-J_{X_{t}}^{\Pi_{t}} J_{\Pi_{t}}{ }^{-1} J_{\Pi_{t}}^{X_{t}}\right]^{-1}
$$


As a uniform a priori is assumed for the $\Pi_{t}$ law, $J_{\Pi_{t+1}}^{\Pi_{t+1}}\left(p\left(\Pi_{t+1}\right)\right)$ is null. To evaluate the third term of $D_{\Phi_{t}}^{22}$, we can write:

$$
\begin{aligned}
& \log p\left(Y_{t}=y_{t} \mid \Phi_{t}=\phi_{t}\right) \stackrel{A 1}{=}={ }^{A 3} \log \prod_{j=1}^{m_{t}} p\left(y_{t}^{j} \mid \phi_{t}\right)= \\
& \sum_{j=1}^{m_{t}} \log \left(\frac{\pi_{t}^{0}}{V}-\pi_{t}^{0} p\left(y_{t}^{j} \mid x_{t}^{M}\right)+\sum_{i=1}^{M-1}\left(p\left(y_{t}^{j} \mid x_{t}^{i}\right)-p\left(y_{t}^{j} \mid x_{t}^{M}\right)\right) \pi_{t}^{i}+p\left(y_{t}^{j} \mid x_{t}^{M}\right)\right) .
\end{aligned}
$$

For $i \neq M$, the gradient w.r.t. $X_{t}^{i}$ is:

$$
\nabla_{X_{t}^{i}} \log p\left(y_{t} \mid \phi_{t}\right)=\pi_{t}^{i} \sum_{j=1}^{m_{t}} \frac{\nabla_{X_{t}^{i}} p\left(y_{t}^{j} \mid x_{t}^{i}\right)}{p\left(y_{t}^{j} \mid \phi_{t}\right)}
$$

and the same expression for $i=M$ replacing $\pi_{t}^{M}$ by $1-\sum_{i=0}^{M-1} \pi_{t}^{i}$. For $i=1, \ldots, M-1$ :

$$
\nabla_{\Pi_{t}^{i}} \log p\left(y_{t} \mid \phi_{t}\right)=\sum_{j=1}^{m_{t}} \frac{p\left(y_{t}^{j} \mid x_{t}^{i}\right)-p\left(y_{t}^{j} \mid x_{t}^{M}\right)}{p\left(y_{t}^{j} \mid \phi_{t}\right)} .
$$

After some computations, we obtain for $i 1, i 2 \neq M$

$$
\begin{aligned}
& J_{X_{t}^{i 1}}^{X_{t}^{i 2}}\left(p\left(Y_{t} \mid \Phi_{t}\right)\right) \triangleq \mathbb{E}\left[\nabla_{X_{t}^{i 1}}\left(\nabla_{X_{t}^{i 2}} \log p\left(Y_{t} \mid \Phi_{t}\right)\right)^{T}\right]= \\
& \mathbb{E}_{\Phi_{t}}\left[\pi_{t}^{i 1} \pi_{t}^{i 2} \sum_{j=1}^{m_{t}} \mathbb{E}_{Y_{t}^{j} \mid \Phi_{t}} \frac{\nabla_{X_{t}^{i 1}} p\left(y_{t}^{j} \mid x_{t}^{i 1}\right)\left(\nabla_{X_{t}^{i 2}} p\left(y_{t}^{j} \mid x_{t}^{i 2}\right)\right)^{T}}{p\left(y_{t}^{j} \mid \phi_{t}\right)^{2}}\right] ;
\end{aligned}
$$

and the same expressions for $i 1$ or $i 2=M$ by replacing $\pi_{t}^{M}$ by $1-\sum_{i=0}^{M-1} \pi_{t}^{i}$.

For $i 1, i 2 \neq M$ :

$$
J_{\Pi_{t}^{i 1}}^{\Pi_{i}^{i 2}}\left(p\left(Y_{t} \mid \Phi_{t}\right)\right)=\mathbb{E}_{\Phi_{t}}\left[\sum_{j=1}^{m_{t}} \mathbb{E}_{Y_{t}^{j} \mid \Phi_{t}}\left[\frac{\left(p\left(y_{t}^{j} \mid x_{t}^{i 1}\right)-p\left(y_{t}^{j} \mid x_{t}^{M}\right)\right)\left(p\left(y_{t}^{j} \mid x_{t}^{i 2}\right)-p\left(y_{t}^{j} \mid x_{t}^{M}\right)\right)}{p\left(y_{t}^{j} \mid \phi_{t}\right)^{2}}\right]\right] .
$$

For $i 1, i 2 \neq M$ :

$$
J_{X_{t}^{i 1}}^{\Pi_{t}^{i 2}}\left(p\left(Y_{t} \mid \Phi_{t}\right)\right)=\mathbb{E}_{\Phi_{t}}\left[\pi_{t}^{i 1} \sum_{j=1}^{m_{t}} \mathbb{E}_{Y_{t}^{j} \mid \Phi_{t}}\left[\frac{p\left(y_{t}^{j} \mid x_{t}^{i 2}\right)-p\left(y_{t}^{j} \mid x_{t}^{M}\right)}{p\left(y_{t}^{j} \mid \phi_{t}\right)^{2}} \nabla_{X_{t}^{i 1}} p\left(y_{t}^{j} \mid x_{t}^{i 1}\right)\right]\right] ;
$$

and the same expressions for $i 1=M$ by replacing $\pi_{t}^{M}$ by $1-\sum_{i=0}^{M-1} \pi_{t}^{i}$.

Notice that under these association assumptions, all the integral w.r.t. $y_{t}^{j}$ are $n_{y}$ dimensional.

\subsection{PCRB B3}

The association vector is supposed to be known. We then have:

$$
\log p\left(Y_{t}=y_{t} \mid X_{t}=x_{t}, K_{t}=k_{t}\right)=\sum_{j=1}^{m_{t}} \log p\left(y_{t}^{j} \mid x_{t}^{k_{t}^{j}}\right) .
$$

The gradient of the log-likelihood w.r.t. $X_{t}^{i}$ is not null only if there exists $j^{i}$ such that $k_{t}^{j}=i$. In this case,

$$
\nabla_{X_{t}^{i}} \log p\left(y_{t} \mid x_{t}, k_{t}\right)=\frac{\nabla_{X_{t}^{i}} p\left(y_{t}^{j^{i}} \mid x_{t}^{i}\right)}{p\left(y_{t}^{j^{i}} \mid x_{t}^{i}\right)}
$$


We finally obtain for all $i, \ldots, M$ :

$$
\begin{gathered}
J_{X_{t}^{i}}^{X_{t}^{i}}\left(p\left(y_{t} \mid x_{t}, k_{t}\right)\right)=\mathbb{E}_{X_{t}} \mathbb{E}_{Y_{t}^{j^{i}} \mid X_{t}} \frac{\nabla_{X_{t}^{i}} p\left(y_{t}^{j^{i}} \mid x_{t}^{i}\right)\left(\nabla_{X_{t}^{i}} p\left(y_{t}^{j^{i}} \mid x_{t}^{i}\right)\right)^{T}}{p\left(y_{t}^{j^{i}} \mid x_{t}^{i}\right)^{2}} \\
\text { and, } J_{X_{t}^{i_{i}^{i}}}^{X_{i 2}^{i 2}}\left(p\left(y_{t} \mid x_{t}, k_{t}\right)\right)=0 \text { if } i 1 \neq i 2 .
\end{gathered}
$$

\subsection{Monte Carlo evaluation in the bearings-only application}

In this case, the evolution model, given by (7), is linear and Gaussian. We analytically obtain, the following equalities: $D_{X_{t}}^{11}=\operatorname{diag}\left\{F^{i^{T}} \Sigma_{V}^{-1} F^{i}\right\}$, $1 D_{X_{t}}^{12}=\operatorname{diag}\left\{-F^{i^{T}} \Sigma_{V}^{-1}\right\}$ and $J_{X_{t+1}}^{X_{t+1}}\left(p\left(X_{t+1} \mid X_{t}\right)\right)=\operatorname{diag}\left\{\Sigma_{V}^{-1}\right\}$. In the general case of an observation model with an additive gaussian noise defined as follows:

$$
\begin{aligned}
& p\left(y_{t}^{j} \mid x_{t}^{i}\right)=\left(\pi^{n_{y}} \operatorname{det} \Sigma\right)^{-\frac{1}{2}} \exp \left\{-\frac{1}{2}\left(y_{t}^{j}-H\left(x_{t}^{i}\right)\right)^{T} \Sigma^{-1}\left(y_{t}^{j}-H\left(x_{t}^{i}\right)\right)\right\} \\
& \text { we have } \nabla_{X_{t}^{i 1}} p\left(y_{t}^{j} \mid x_{t}^{i 1}\right)=p\left(y_{t}^{j} \mid x_{t}^{i 1}\right) \nabla_{X_{t}^{i 1}} H^{T}\left(x_{t}^{i 1}\right) \Sigma^{-1}\left(y_{t}^{j}-H\left(x_{t}^{i 1}\right)\right) .
\end{aligned}
$$

It reads for the PCRB B1:

$$
\begin{aligned}
& J_{X_{t}^{i 1}}^{X_{t}^{i 2}}\left(p\left(Y_{t} \mid X_{t}\right)\right)=\mathbb{E}_{X_{t}}\left[\nabla_{X_{t}^{i 1}} H^{T}\left(x_{t}^{i 1}\right) \Sigma^{-1}\right. \\
& \left.\mathbb{E}_{Y_{t} \mid X_{t}}\left[\frac{\sum_{k_{t} \supset i 1} p\left(y_{t} \mid x_{t}, k_{t}\right) p\left(k_{t}\right)\left(y_{t}^{j^{i 1}}-H\left(x_{t}^{i 1}\right)\right)}{p\left(y_{t} \mid x_{t}\right)^{2}} \cdot \sum_{k_{t} \supset i 2} p\left(y_{t} \mid x_{t}, k_{t}\right) p\left(k_{t}\right)\left(y_{t}^{j^{i 2}}-H\left(x_{t}^{i 2}\right)\right)^{T}\right] \Sigma^{-1}\left(\nabla_{X_{t}^{i 2}} H^{T}\left(x_{t}^{i 2}\right)\right)^{T}\right] ;
\end{aligned}
$$

for the PCRB B2:

$$
\begin{aligned}
& J_{X_{t}^{i 1}}^{X_{t}^{i 2}}\left(p\left(Y_{t} \mid \Phi_{t}\right)\right)=\mathbb{E}_{\Phi_{t}}\left[\pi_{t}^{i 1} \pi_{t}^{i 2} \nabla_{X_{t}^{i 1}} H^{T}\left(x_{t}^{i 1}\right) \Sigma^{-1} \sum_{j=1}^{m_{t}} \mathbb{E}_{Y_{t}^{j} \mid \Phi_{t}}\left[\frac{p\left(y_{t}^{j} \mid x_{t}^{i 1}\right) p\left(y_{t}^{j} \mid x_{t}^{i 2}\right)}{p\left(y_{t}^{j} \mid \phi_{t}\right)^{2}}\left(y_{t}^{j}-H\left(x_{t}^{i 1}\right)\right)\left(y_{t}^{j}-H\left(x_{t}^{i 2}\right)\right)^{T}\right]\right. \\
& \left.\Sigma^{-1}\left(\nabla_{X_{t}^{i 2}} H^{T}\left(x_{t}^{i 2}\right)\right)^{T}\right] . \\
& J_{X_{t}^{i 1}}^{\Pi_{i}^{i 2}}\left(p\left(Y_{t} \mid \Phi_{t}\right)\right)=\mathbb{E}_{\Phi_{t}}\left[\pi_{t}^{i 1} \nabla_{X_{t}^{i 1}} H^{T}\left(x_{t}^{i 1}\right) \Sigma^{-1} \sum_{j=1}^{m_{t}} \mathbb{E}_{Y_{t}^{j} \mid \Phi_{t}}\left[\frac{\left.p\left(y_{t}^{j} \mid x_{t}^{i 2}\right)-p\left(y_{t}^{j} \mid x_{t}^{M}\right)\right)}{p\left(y_{t}^{j} \mid \phi_{t}\right)^{2}} p\left(y_{t}^{j} \mid x_{t}^{i 1}\right)\left(y_{t}^{j}-H\left(x_{t}^{i 1}\right)\right)\right]\right] .
\end{aligned}
$$

and for the PCRB B3:

$$
J_{X_{t}^{i}}^{X_{i}^{i}}\left(p\left(Y_{t} \mid X_{t}\right)\right)=\mathbb{E}_{X_{t}^{i}} \nabla_{X_{t}^{i}} H^{T}\left(x_{t}^{i}\right) \Sigma^{-1}\left(\nabla_{X_{t}^{i}} H^{T}\left(x_{t}^{i}\right)\right)^{T} .
$$

In the bearings-only application, we have $n_{y}=1$ and then $H^{T}=H$ that leads to some writing simplifications.

The three bounds are first initialized to $J_{X_{0}}=P_{X_{0}}^{-1}$ for B1 and B3 and $J_{\Phi_{0}}=P_{\Phi_{0}}^{-1}$ for $B 2$ where $P_{X_{0}}=$ $\operatorname{diag}\left\{X_{\text {cov }}^{i}\right\}$ and $X_{c o v}^{i}$ are the covariance matrices defined in (11), and $P_{\Phi_{0}}=\operatorname{diag}\{\operatorname{diag}\{0.05, i=1, \ldots, M-$ $\left.1\} ; P_{X_{0}}\right\}$. Then, to estimate the matrices needed in the recursion formulas (15) or (26), we perform Monte Carlo integration by carrying out $P 1$ independent state trajectories and for each of them $P 2$ independent

\footnotetext{
${ }^{1}$ i.e. the block-diagonal matrix whose $i$ th block is equal to $F^{i T} \Sigma_{V}^{-1} F^{i}$
} 

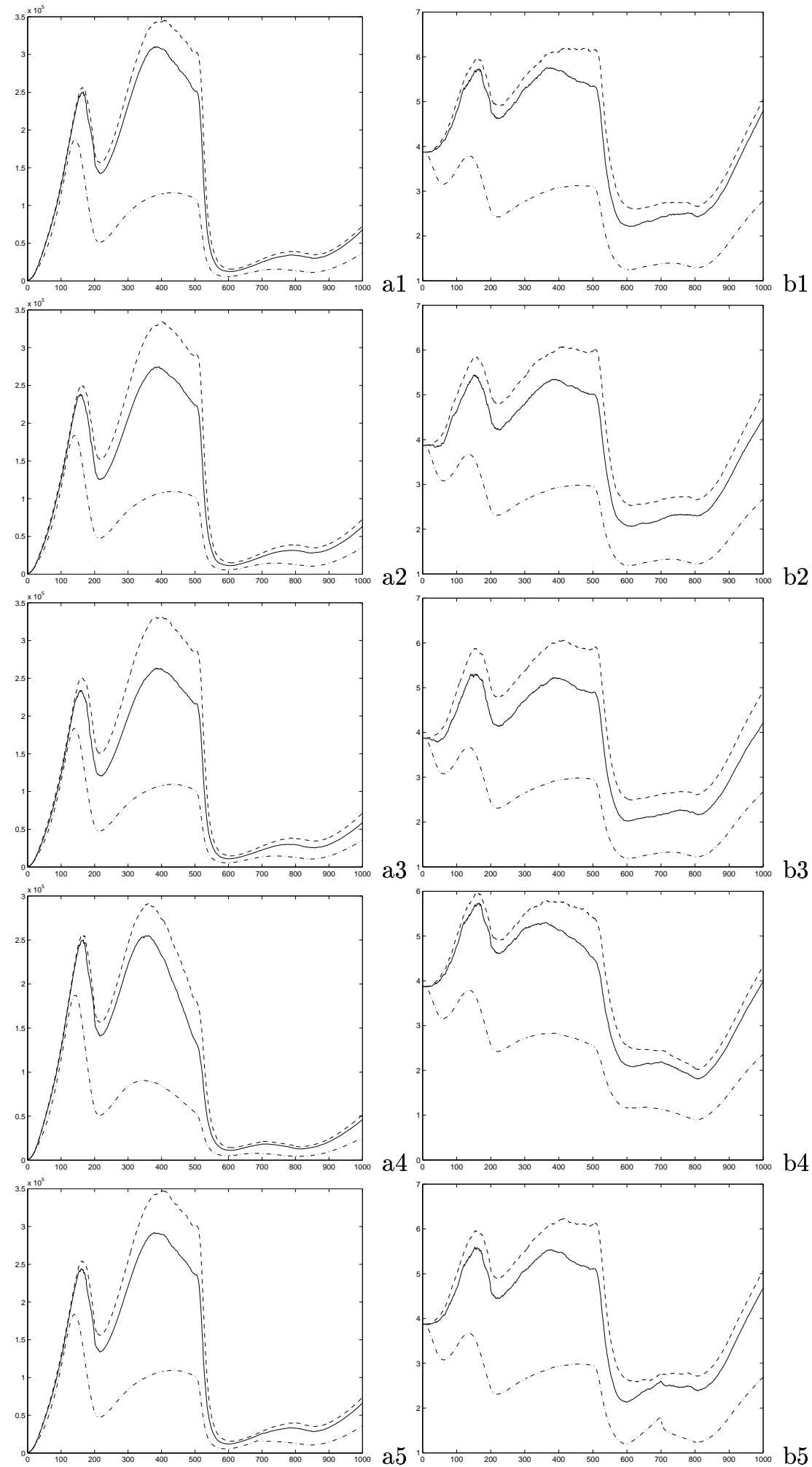

Figure 4: Comparison of the trace and of the volume of the three matrices B1 (dashed), B2 (solid), and B3 (dashdot). First column: trace, Second column: volume. First row: $\sigma_{x}=\sigma_{y}=0.0005 \mathrm{~ms}^{-1}$ and $\lambda V=1$, Second row: $\sigma_{x}=\sigma_{y}=0.0005 \mathrm{~ms}^{-1}$ and $\lambda V=2$, Third row: $\sigma_{x}=\sigma_{y}=0.0005 \mathrm{~ms}^{-1}$ and $\lambda V=3$, Fourth row: $\sigma_{x}=\sigma_{y}=0.0001 \mathrm{~ms}^{-1}$, and $\lambda V=1$, Fifth row: $\sigma_{x}=\sigma_{y}=0.0005 \mathrm{~ms}^{-1}$ and a detection hole between times 600 and 700 for object 1 . 
measurement realizations, and additionally $P 3$ independent realizations of the $\pi$ vector for the PCRB B2. For instance, the estimate $\hat{J}_{X_{t}^{i 1}}^{X_{i 1}^{i 2}}$ of $J_{X_{t}^{i 1}}^{X_{t}^{i 2}}$ is computed as:

$$
\hat{J}_{X_{t}^{i 1}}^{X_{t}^{i 2}}=\frac{1}{P 1 P 2} \sum_{p 1=1}^{P 1} \sum_{p 2=1}^{P 2} J\left(x_{t}^{p 1}, y_{t}^{p 1, p 2}\right)
$$

where $J\left(x_{t}^{p 1}, y_{t}^{p 1, p 2}\right)$ is the quantity whose expectation is to be computed in (41). We then obtained the matrix inequalities:

$$
\mathbb{E}\left(\hat{X}_{t+1}^{1: M}(Y)-X\right)\left(\hat{X}_{t+1}^{1: M}(Y)-X\right)^{T} \geq B^{i} \text { for } i=1,2,3 .
$$

In the following, we have considered the target and observations models exposed in 3.1 with the same observer trajectories and initial targets positions. The matrices $B^{i}$ dimension is then equal to $\operatorname{dim}=3 \times 4=12$. To interpret the inequalities (46), we have derived the scalar mean-square error given by the trace of (46):

$$
\mathbb{E}\left(\hat{X}_{t+1}^{1: M}(Y)-X\right)^{T}\left(\hat{X}_{t+1}^{1: M}(Y)-X\right) \geq \operatorname{tr} B^{i}
$$

and the inequality on the volume of the matrices defined as the determinant at the power $1 /$ dim:

$$
\left[\operatorname{det} \mathbb{E}\left(\hat{X}_{t+1}^{1: M}(Y)-X\right)\left(\hat{X}_{t+1}^{1: M}(Y)-X\right)^{T}\right]^{1 / \operatorname{dim}} \geq\left[\operatorname{det} B^{i}\right]^{1 / \operatorname{dim}}
$$

We have computed the trace and the volume of the three bounds for different values of the parameters $\sigma_{x}, \sigma_{y}, P_{d}, \lambda V$. First, for a dynamic noise std $\sigma_{x}=\sigma_{y}=0.0005 \mathrm{~ms}^{-1}$, a detection probability $P_{d}=0.9$ and $\lambda V=1,2,3$, the trace and the volume are plotted against time on the three first rows of Fig 4 The results on the fourth row have been obtained for a higher dynamic noise std $\sigma_{x}=\sigma_{y}=0.001 \mathrm{~ms}^{-1}, P_{d}=0.9$ and $\lambda V=1$. The fifth and last row corresponds to a scenario where a detection hole is simulated for the first object during hundred consecutive instants, between times 600 and 700 . Whatever the parameters values, the instant or the function $f$ of the bounds considered (trace or volume), we always have: $f(B 1) \geq f(B 2) \geq f(B 3)$ with a greater gap between $f(B 2)$ and $f(B 3)$ than between $f(B 1)$ and $f(B 2)$. More precisely, it first means that the optimal performance which can be obtained with an algorithm using assumptions (A1) and (A2) are below the optimal performance which can be obtained with an algorithm using assumptions (A1) and (A3). Second, the optimal performance obtained with an algorithm assuming the association is known are far better than for the two preceding cases. For all that, nothing can be concluded on the relative performance of the SIR-JPDA and of the MOPF. Such study needs the estimation of the mean-square error of both algorithms over a high number of realizations of the process and measurement noise. For each couple of realization of both noises, several runs of the algorithms are needed as it has been done in Section 3 for one particular realization. To go back over the analysis of Fig 4 the plots present two peaks around times 150 and 400 . They correspond to instants where bearings from the three targets are very close as shown in Fig 2 b for one particular realization of the trajectories and of the measurements. During the second peak, the gap between B1 and B2 on the one hand and B3 on the other hand is widening. A light peak is also observed when the first target is not detected (see last row of Fig 4). Finally, by comparing the three first rows, we observe that the gap between $f(B 1)$ and $f(B 2)$ is widening with the clutter density $\lambda V$.

In all these scenarios, as the detection probability $P_{d}$ is strictly inferior to unity, it may happen at one instant that no target is detected. If moreover no clutter measurement is simulated at that instant, the measurement vector $Y_{t}$ is empty. In this case, we simply set the expectations $J_{X_{t+1}}^{X_{t+1}}\left(p\left(Y_{t+1} \mid X_{t+1}\right)\right)$ and $J_{\Phi_{t+1}}^{\Phi_{t+1}}\left(p\left(\Phi_{t+1} \mid X_{t+1}\right)\right)$ to zero and the recursive formula (15) and (26) are reduced.

\section{Conclusion}

Two sequential Monte Carlo methods for multi-target tracking have been compared for a classical bearingsonly application. Then, the extension of the posterior Cramér-Rao bound from single to multi-target filtering problem has been studied. Three bounds have been derived according to the association assumptions between the measurements and the targets. Based on Monte Carlo integration, estimates of these three bounds have finally been proposed and evaluated for the bearings-only application. 


\section{References}

[1] T. E. Fortmann, Y. Bar-Shalom, and M. Scheffe. Sonar tracking of multiple targets using joint probabilistic data association. IEEE Journal of Oceanic Engineering, 8:173-184, July 1983.

[2] D. Reid. An algorithm for tracking multiple targets. IEEE Transactions on Automation and Control, 24(6):84-90, 1979.

[3] H. Gauvrit, J.-P. Le Cadre, and C. Jauffret. A formulation of multitarget tracking as an incomplete data problem. IEEE Trans. on Aerospace and Electronic Systems, 33(4):1242-1257, October 1997.

[4] R. L. Streit and T. E. Luginbuhl. Maximum likelihood method for probabilistic multi-hypothesis tracking. In Proceedings of SPIE International Symposium, Signal and Data Processing of Small Targets 1994, volume 2235, Orlando, FL, 5-7 April 1994.

[5] Y. Bar-Shalom and T.E. Fortmann. Tracking and data association. Academic Press, 1988.

[6] C. Hue, J.-P. Le Cadre, and P. Pérez. Sequential Monte Carlo methods for multiple target tracking and data fusion. IEEE Trans. on Signal Processing, 50(2):309-325, February 2002.

[7] R. Karlsson and F. Gustafsson. Monte Carlo data association for multiple target tracking. In IEE Seminar - Target Tracking: algorithms and applications, pages 13/1-13/5, 16-17 october 2001.

[8] M. Orton and A. Marrs. A Bayesian approach to multi-target tracking and data fusion with out-ofsequence measurements. In IEE Seminar - Target Tracking: algorithms and applications, pages 15/1$15 / 5,16-17$ october 2001.

[9] H. L. Van Trees. Detection, Estimation, and Modulation Theory (Part I). John Wiley and Sons, 1968.

[10] P. Tichavsky, C. Muravchik, and A. Nehorai. Posterior Cramér-Rao bounds for discrete-time nonlinear filtering. IEEE Trans. Signal Processing, 46(5):1386-1396, May 1998.

[11] N. Bergman. Recursive Bayesian estimation: navigation and tracking applications. PhD thesis, Linköping University, Sweden, 1999.

[12] X. Zhang and P. Willett. Cramér-Rao bounds for discrete-time linear filtering with measurement origin uncertainties. In Workshop on Estimation, Tracking, and Fusion: A Tribute to Yaakov Bar-Shalom, May 2001.

[13] M. Hernandez, A. Marrs, N. Gordon, S. Maskell, and C. Reed. Cramér-Rao bounds for nonlinear filtering with measurement origin uncertainty. In 5th International Conference on Information Fusion, July 2002.

\section{Appendix: recursive formula of $\mathrm{PCRB} \mathrm{B} 2$}

By definition, the information matrix $J\left(\Phi_{0: t+1}\right)$ of $\Phi_{0: t+1}$ associated with the law $p_{t+1}$ can be expressed as:

$$
J\left(\Phi_{0: t-1}\right) \triangleq\left[\begin{array}{ccc}
J_{\Phi_{0: t-1}}^{\Phi_{0: t-1}}\left(p_{t+1}\right) & J_{\Phi_{0: t-1}}^{\Phi_{t}}\left(p_{t+1}\right) & J_{\Phi_{0: t-1}}^{\Phi_{t+1}}\left(p_{t+1}\right) \\
J_{\Phi_{0: t-1}}^{\Phi_{t}}\left(p_{t+1}\right) & J_{\Phi_{t}}^{\Phi_{t}}\left(p_{t+1}\right) & J_{\Phi_{t}}^{\Phi_{t+1}}\left(p_{t+1}\right) \\
J_{\Phi_{t+1}}^{\Phi_{0: t-1}}\left(p_{t+1}\right) & J_{\Phi_{t+1}}^{\Phi_{t}}\left(p_{t+1}\right) & J_{\Phi_{t+1}}^{\Phi_{t+1}}\left(p_{t+1}\right)
\end{array}\right]
$$

where $J_{\alpha}^{\beta}(p) \triangleq \mathbb{E}\left[-\Delta_{\alpha}^{\beta} \log (p)\right]$. Using (24), it reads:

$$
J_{\Phi_{0: t-1}}^{\Phi_{0: t-1}}\left(p_{t+1}\right)=J_{\Phi_{0: t-1}}^{\Phi_{0: t-1}}\left(p_{t}\right)+\underbrace{\left.J_{\Phi_{0: t-1}}^{\Phi_{0: t-1}}\left(p\left(Y_{t+1} \mid \Phi_{t+1}\right) p\left(X_{t+1} \mid X_{t}\right) p\left(\Pi_{t+1}\right)\right)\right)}_{=0} ;
$$




$$
\begin{aligned}
& J_{\Phi_{t}}^{\Phi_{0: t-1}}\left(p_{t+1}\right)=J_{\Phi_{t}}^{\Phi_{0: t-1}}\left(p_{t}\right)+\underbrace{\left.J_{\Phi_{t}}^{\Phi_{0: t-1}}\left(p\left(Y_{t+1} \mid \Phi_{t+1}\right) p\left(X_{t+1} \mid X_{t}\right) p\left(\Pi_{t+1}\right)\right)\right)}_{=0} ; \\
& J_{\Phi_{t+1}}^{\Phi_{0: t-1}}\left(p_{t+1}\right)=\underbrace{J_{\Phi_{t+1}}^{\Phi_{0: t}}\left(p_{t}\right)}_{=0}+\underbrace{\left.J_{\Phi_{t}}^{\Phi_{0: t-1}}\left(p\left(Y_{t+1} \mid \Phi_{t+1}\right) p\left(X_{t+1} \mid X_{t}\right) p\left(\Pi_{t+1}\right)\right)\right)}_{=0} ; \\
& J_{\Phi_{t}}^{\Phi_{t}}\left(p_{t+1}\right)=J_{\Phi_{t}}^{\Phi_{t}}\left(p_{t}\right)+J_{\Phi_{t}}^{\Phi_{t}}\left(p\left(X_{t+1} \mid X_{t}\right)\right)+\underbrace{\left.J_{\Phi_{t}}^{\Phi_{t}}\left(p\left(Y_{t+1} \mid \Phi_{t+1}\right) p\left(\Pi_{t+1}\right)\right)\right)}_{=0} ; \\
& J_{\Phi_{t}}^{\Phi_{t+1}}\left(p_{t+1}\right)=J_{\Phi_{t}}^{\Phi_{t+1}}\left(p\left(X_{t+1} \mid X_{t}\right)\right)+\underbrace{J_{\Phi_{t}}^{\Phi_{t+1}}\left(p_{t}\right)}_{=0}+\underbrace{\left.J_{\Phi_{t}}^{\Phi_{t+1}}\left(p\left(Y_{t+1} \mid \Phi_{t+1}\right) p\left(\Pi_{t+1}\right)\right)\right)}_{=0} ; \\
& J_{\Phi_{t+1}}^{\Phi_{t+1}}\left(p_{t+1}\right)=\underbrace{J_{\Phi_{t+1}}^{\Phi_{t+1}}\left(p_{t}\right)}_{=0}+J_{\Phi_{t+1}}^{\Phi_{t+1}}\left(p\left(Y_{t+1} \mid \Phi_{t+1}\right) p\left(X_{t+1} \mid X_{t}\right) p\left(\Pi_{t+1}\right)\right)
\end{aligned}
$$

Using (50)-(55) and the notation:

$$
J\left(\Phi_{0: t}\right)=\left[\begin{array}{cc}
A_{t} & B_{t} \\
B_{t}^{T} & C_{t}
\end{array}\right]
$$

we have the recursive formula:

$$
J\left(\Phi_{0: t+1}\right)=\left[\begin{array}{ccc}
A_{t} & B_{t} & 0 \\
B_{t}^{T} & C_{t}+D_{t}^{11} & D_{t}^{12} \\
0 & D_{t}^{12^{T}} & D_{t}^{22}
\end{array}\right]
$$

where

$$
\begin{aligned}
& D_{t}^{11}=J_{\Phi_{t}}^{\Phi_{t}}\left(p\left(X_{t+1} \mid X_{t}\right)\right) \\
& D_{t}^{12}=J_{\Phi_{t}}^{\Phi_{t+1}}\left(p\left(X_{t+1} \mid X_{t}\right)\right) \\
& D_{t}^{22}=J_{\Phi_{t+1}}^{\Phi_{t+1}}\left(p\left(Y_{t+1} \mid \Phi_{t+1}\right) p\left(X_{t+1} \mid X_{t}\right) p\left(\Pi_{t+1}\right)\right) .
\end{aligned}
$$

Now, $J_{\Phi_{t+1}}$ is the inverse of the right lower block of $J\left(\Phi_{0: t+1}\right)^{-1}$. Using twice a classical inversion lemma, we obtain:

$$
\begin{array}{r}
J_{\Phi_{t+1}}=D_{t}^{22}-\left[\begin{array}{ll}
0 & D_{t}^{12}
\end{array}\right]\left[\begin{array}{cc}
A_{t} & B_{t} \\
B_{t}^{T} & C_{t}+D_{t}^{11}
\end{array}\right]^{-1}\left[\begin{array}{c}
0 \\
D_{t}^{12}
\end{array}\right] \\
=D_{t}^{22}-D_{t}^{12}\left[C_{t}+D_{t}^{11}-B_{t}^{T} A_{t}^{-1} B_{t}\right]^{-1} D_{t}^{12} \\
=D_{t}^{22}-D_{t}^{12}\left[J_{\Phi_{t}}+D_{t}^{11}\right]^{-1} D_{t}^{12}
\end{array}
$$




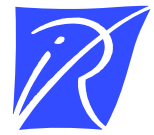

Unité de recherche INRIA Rennes

IRISA, Campus universitaire de Beaulieu - 35042 Rennes Cedex (France)

Unité de recherche INRIA Lorraine : LORIA, Technopôle de Nancy-Brabois - Campus scientifique 615, rue du Jardin Botanique - BP 101 - 54602 Villers-lès-Nancy Cedex (France)

Unité de recherche INRIA Rhône-Alpes : 655, avenue de l'Europe - 38330 Montbonnot-St-Martin (France)

Unité de recherche INRIA Rocquencourt : Domaine de Voluceau - Rocquencourt - BP 105 - 78153 Le Chesnay Cedex (France)

Unité de recherche INRIA Sophia Antipolis : 2004, route des Lucioles - BP 93 - 06902 Sophia Antipolis Cedex (France) 